Z. klin. Chem. u. klin. Biochem.

8. Jg., S. 564-566, November 1970

\title{
Die Entwicklung der Speichel- und Pankreas-Amylase bei Kindern im Laufe des ersten Lebensjahres ."
}

\author{
Von J. Kamarít und Olga Fintajslová \\ Aus dem Forscbungsinstitut für Pädiatrie (Direktor: Prof. MUDr. Z. Brunecký, C. Sc.), Brno (ČCSR्) und der

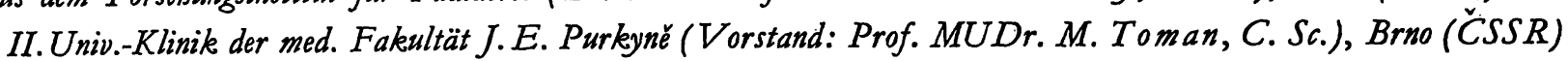

(Eingegangen am 16. Juni 1970)

Die mit Agar-Gel-Elektrophorese fraktionierte $\alpha$-Amylase des Blutserums von Kindern im Laufe des ersten Lebensjahres zeigte im ersten Lebensmonat nur Aktivitäten von Speichelamylase (2-5 STREET-CLOSE-Einheiten); eine Aktivität der pankreatischen Amylase war nicht nachwcisbar. Die Aktivitäten beider Amylasen nehmen fortschreitend zu und am Ende des ersten Lebensjahres ist die Speichelamylase $\mathrm{mit}^{2} / 3$ und die Pankreas-Amylase mit $1 / 3$ an der Gesamtaktivität (7-15 E.) beteiligt. Die Unregelmäßigkeit der steigenden Kurve der beiden Aktivitäten ist wahrscheinlich auf die beginnende Dentitio zurückzuführen. Im Vergleich mit den Erwachsenen ist die hohe Schweiß-Amylase-Aktivität bei diesen Kindern seht interessant. Auch auf die hohen Amylase-Aktivitäten in der Muttermilch wird hier hingewiesen; die Bedeutung dieses Enzymes wird diskutiert.

\section{The development of salivary and pancreatic amylase in children during the first year of life}

The $\alpha$-amylase from the serum of children during the first year of life was fractionated by agar gel electrophoresis. In the first month, the salivary' amylase activity was 2-5 STREET-CLOSE units and pancreatic amylase activity was not measurable. Both activities increase progressively and at the end of the first year the total activity is 7-15 STREET-CLOSE units, of which two thirds is salivary amylase and one third pancreatic amylase. The two curves of increasing activity show a irregularity, probably as a result of teething. The high perspiration amylase activity of these children in comparison with adults is interesting. In this connection the significance of the high amylase activity of mother's milk is discussed.

$\alpha$-Amylase ( $\alpha$-1,4-Glucan 4-glucanohydrolase EC 3.2.1.1), welche durch Speicheldrüsen und Pankreas produziert wird, stellt ein typisches induziertes Enzym dar. Ihre Aktivitäten sind bei den Säuglingen kaum meßbar oder sehr gering und treten fortschreitend erst als Folge der Stärkezufuhr in der Nahrung auf. Infolge des Übertritts von Amylase der Speicheldrüsen und des Pankreas ins Blut kann man im Blutserum beide Amylase-Aktivitäten bestimmen. Bei Erwachsenen bleiben diese Blutserum-Aktivitäten unter physiologischen Umständen auffallend stabil. Ungefähr 0,1\% der AmylaseProduktion der Speicheldrüsen und $0,2-0,25 \%$ des Pankreas geht ins Blut über. Die Nieren-Clearance der beiden Isoamylasen, wovon die Speichel-Amylase nur die Hälfte der Pankreas-Amylase beträgt, stellt dafür auch einen Beweis dar.

Die erwähnten Angaben wurden teils mit Hilfe der elektrophoretischen Trennung der Blutserumamylase, durch Bestimmung der Gesamtamylase und der einzelnen Isoamylase-Aktivitäten, teils durch Bestimmung der Speichel-, Duodenalsaft- und Harnaktivität festgestellt $(1,2,3)$.

Im Laufe der bisherigen Untersuchungen haben wir sieben autosomalkodominant hereditäre Varianten der Amylase gefunden und $z$ war mit einer Frequenz in der Population, welche einen neuen Polymorphismus darstellt $(4,5)$.

Auf Grund der bisherigen Erkenntnisse und methodischen Möglichkeiten haben wir uns die Aufgabe gestellt, die Entwicklung der Aktivität der Speichel- und Pankreas-Amylase bei den Kindern im Laufe des ersten Lebensjahres mit Hilfe der Aktivitätsbestimmung im Blutserum zu ermitteln.

\section{Material und Methodik}

Die Enzym-Aktivitäten wurden im venösen Blut bestimmt. Kapillar-Blut ist prinzipiell nicht zu verwenden, weil eben bei den Neugeborenen und den Kindern im ersten Lebensjahr eine hohe Ausscheidung von Schweiß-Amylase vorkommt.

Die Trennung der Amylase haben wir mittels Agar-Gel-Elektrophorese mit nachfolgender Densitometrie der Aktivitäten durchgeführt. Diese Methode wurde schon früher beschrieben (2). Die Gesamtamylase-Aktivität wurde nach STREeT und CLOSE bestimmt (6).

\section{Ergebnisse}

Wir haben insgesamt 72 Kinder im Alter vom ersten bis zum zwölften Monat untersucht. Die Kinder wurden dem Alter entsprechend ernährt. Sie wiesen keine nachweisbaren Verdauungsbeschwerden auf. Die Mittelwerte der Gesamtamylase-Aktivitäten und die Anteile der Speichel- und Pankreas-Amylase sind in Abbildung 1 zusammengefaßt. Die offenen Säulen stellen die $\mathrm{Ge}$ samtaktivität mit Standardabweichungen; die quer schraffierten Säulen die Speichelamylase und die längs schraffierten Säulen die Pankreas-Amylase dar. Um dịe Entwicklung der Aktivitäten in der Zeitabhängigkeit zu unterstreichen, häben wir die Säulen mit homologen Angaben durch Linien verbunden. Während in den 


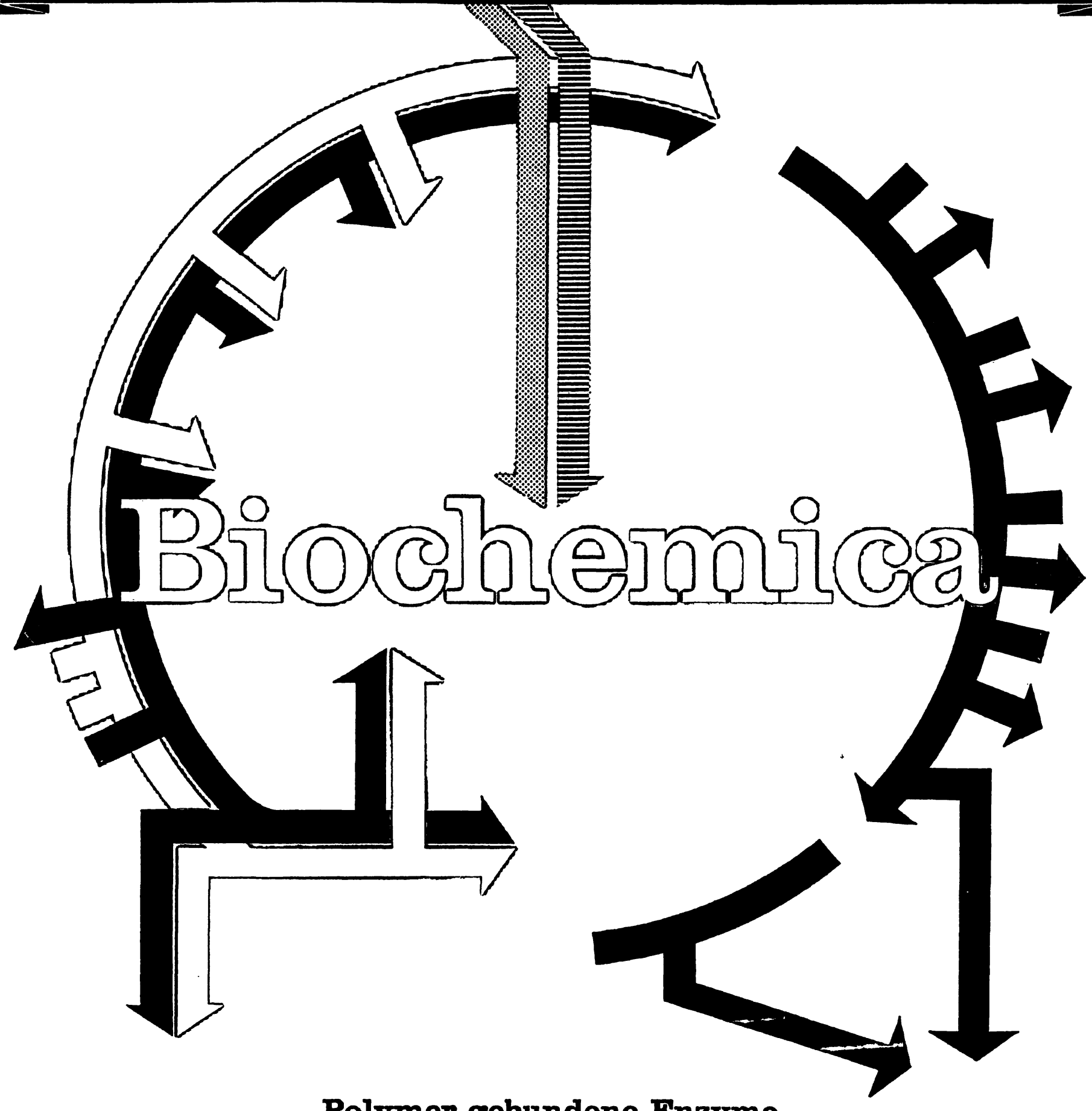

Polymer gebundene Enzyme

Bromelin - Chymotrypsin - Ficin - Papain - Pronase - Proteinase K (Tritirachium album) - Ribonuclease - Subtilisin - Trypsin (Rind) • Trypsin (Schwein) • Trypsin-Inhibitor (Sojabohnen)

\author{
Träger \\ Carboxymethylcellulose - Vernetztes Maleinsäureanhydrid \\ Besondere Vorteile \\ Hohe spezifische Aktivitäten - Enzymaktivität ausschließlich \\ kovalent gebunden - Gleichbleibende Qualität durch \\ Mindestaktivitätsangaben garantiert
}

Ausführliche Spezifikationen stehen auf Wunsch zur Verfügung. 


\section{Jetzł wird Zuverlässigkeit für 2 wichtige diagnostische Methoden geboten}

Bestecks und Reagenzien für die kolorimetrische Bestimmung von

\section{VANILLINMAN DELSÄURE.}

im Harn bei $470-510 \mathrm{~nm}$ mit SIGMA Technical Bulletin Nr. 480.

Die Ausscheidung von Vanillinmandelsäure im Harn ișt bei Patienten mit Phaeochromocytom erhöht (1). SIGMA hat die Methode von Sunderman und Mitarbeitern für die Bestimmung von Vanillinmandelsäure im Harn modifiziert.

Obwohl diese Methode ziemlich kompliziert und zeitraubend ist, erfreut sie sich großer Beliebtheit. Neverdings wurde als nützliche Kontrolle für die Richtigkeit der Ergebnisse ein VanillinmandelsäureStandard in das Verfahren eingeführt.

SIGMA Bulletin Nr. 480 ist auf Anfrage gratis erhältlich.

$\begin{array}{ccc}\text { Order: Besteck-Nr. } & \text { max. mögliche Bestimmungen } & \text { Preis } \\ 480-M & 50 & \$ 21,00 \\ 480-C & 100 & \$ 36,50\end{array}$

Literatur: (1) Sunderman, F. W. Jr., P. D. Cleveland, N. C. Law und F. W. Sunderman, Amer. J. Clin. Path. 34, 293 (1960).

Bestecks und Reagenzien für die kolorimetrische Bestimmung von

\section{7-OXOSTEROIDEN}

im Harn bei 520-560 nm mit SIGMA Technical Bulletin Nr. 17.

Erhöhte Werte von 17-Oxosteroiden im Harn werden bei einer Anzahl von Erkrankungen, z. B. Cushing-Syndrom, Nebennierenstörungen und Makrogenitosomie gefunden. Niedrige Werte kommen vor bei M. Addison, Myxedem und Neophrose.

Prinzip des Tests: die 17-Oxosteroid-Konjugate im Harn werden zuerst mit Säure hydrolysiert. Die freigesetzten Steroide werden mit Diäthyläther extrahiert. Durch Waschen mit Alkali werden Fremdchromogene und Östrogene aus dem Ätherextrakt entfernt.

Die Bestimmung basiert auf der Zimmermann-Reaktion.

SIGMA Bulletin Nr. 17 ist auf Anfrage gratis erhältlich.

Order: Besteck-Nr. max. mögliche Bestimmungen Preis

$17 \quad 100 \quad \$ 14,75$

Es ist ein Vergnügen mit Sigma Geschäfte zu tätigen.

Sigma-Reagenzien sind in der ganzen Welt durch den Fahchandel oder direkt aus St. Louis beziehbar.

Telegramme: SIGMACHEM, St. Louis, Missouri

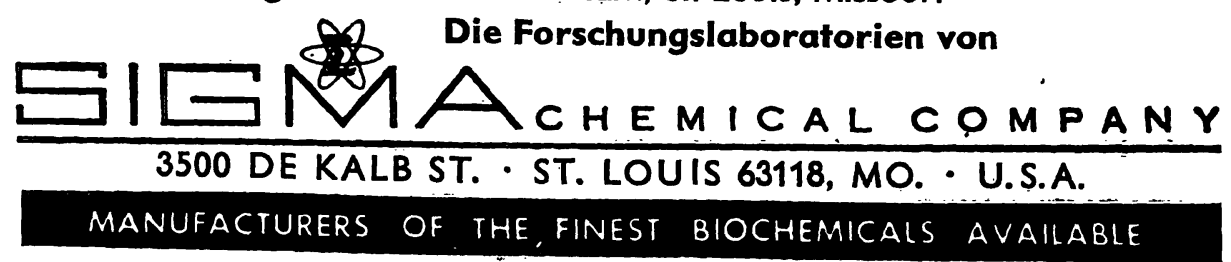

Vertrefung in England: 


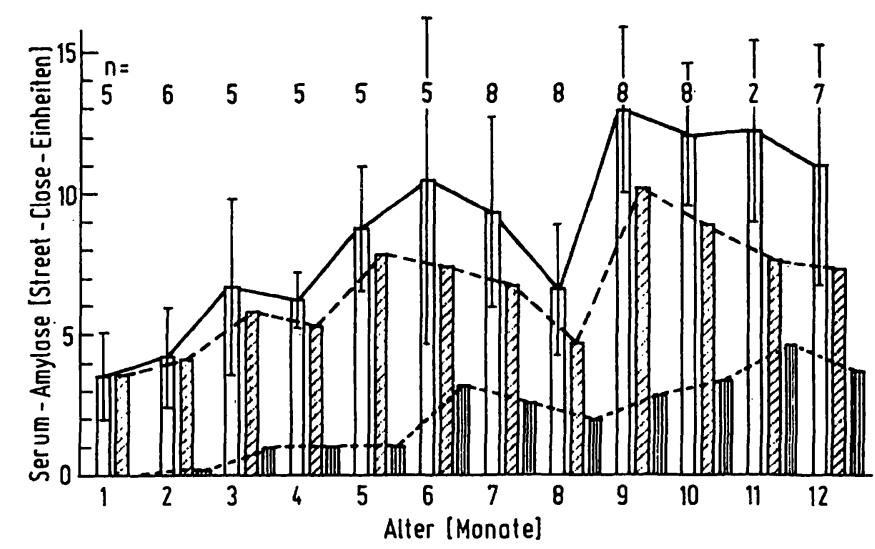

Abb. 1 Die Entwicklung der Blutserum-Amylase-Aktivität im Laufe des

Gesamtaktivität: offene Säulen

Speichelamylase: quer schraffiert

Pankreas-Amylase: längs schraffiert Die Zahlen über den Säulen der Gesamtaktivitäts-Werte stellen die
Zahl der untersuchten Kinder dar

fünf ersten Lebensmonaten die Speichelamylase den Hauptanteil der Gesamtamylase bildet, ist die Pankreasaktivität im ersten Monat nicht meßbar, im zweiten bis fünften Monat erreicht die Aktivität kaum eine ganze Einheit nach STREeT und Close. Ein klarer Anstieg der Pankreas-Amylase-Aktivität beginnt im sechsten Lebensmonat. Im Laufe des ersten Lebensjahres setzt sich die Entwicklung der Aktivitäten so fort, daß am Ende dieser Periode die Gesamtaktivität die Hälfte der Mittelwerte bei Erwachsenen erreicht. Der Speichel-Amylase-Anteil beträgt hier $2 / 3$ und der Pankreas-Amylase-Anteil $1 / 3$ der Gesamtaktivität.

\section{Diskussion}

Beachtenswert in Abbildung 1 ist die Schwankung der Werte in der Periode vom 8. bis 11. Monat und zwar zunächst ein Sinken, dann der Anstieg und endlich wieder ein gewisses Absinken. Obwohl die ermittelten Werte in einzelnen Kindergruppen große Streuungen vorweisen und die untersuchten Gruppen der einzelnen Lebensalter nicht groß sind, geht aus der Abbildung hervor, daß die Schwankungen meistens auf Abweichungen der Speichelamylase beruhen. Bei den meisten Kindern ist jedoch diese Periode identisch mit dem Zeitabschnitt der ersten Dentitio. Es wäre also möglich, diese Schwankung der laufend steigenden Aktivitätskurve auch als Folge des erhöhten Übertritts der Amylase aus der Speicheldrüse in die Blutbahnzu erklären. Das darauf folgende Sinken der Werte könnte man als Dämpfung dieser Funktion charakterisieren. Die auf Grund der Speichelamylase- und Pankreas-AmylaseAktivitäts-Bestimmung im Serum erhaltenen Ergebnisse sind in Übereinstimmung mit den histologischen Befunden von SeIfert, wie sie von KLeINBAum zitiert sind (7). Danach steigt das Gewicht des Pankreas von $3 \mathrm{~g}$ bei der Geburt auf $7 \mathrm{~g}$ am Ende des ersten Lebensjahres. Als Folge der steigenden Nahrungsmengen und vor allem der Qualitätsänderung der Nahrung steigt auch die Funktionsbelastung der Pankreasexkretion.

Was den Amylasepolymorphismus betrifft, so haben wir in der ganzen untersuchten Gruppe nur eine einzige Variante mit zwei pankreatischen Fraktionen und eine Variante mit zwei Speichel-Fraktionen gefunden. Wahrscheinlich kann man den voll entwickelten Phänotyp erst nach Erreichen des ersten bis zweiten Lebensjahres sicher bestimmen. Im Gegensatz $\mathrm{zu}$ den niedrigen Amylase-Aktivitäten bei Neugeborenen steht die hohe Aktivität des amylolytischen Enzyms im Schweiß dieser Kinder. Dieses Enzym weist dieselbe elektrophoretische Beweglichkeit wie die Speichelamylase auf (8). Die Bedeutung dieses Enzyms, welches sich nach HALPRIN und Mitarbeiter (9) im Vergleich zur PhosphorylaseAktivität nur mit ${ }^{1} / 10$ an der Glykogen-Spaltung beteiligt, ist noch nicht ganz klar. Beachtenswert ist auch die hohe Amylase-Aktivität der Muttermilch, in der man Werte von 1200 bis 1700 Street-Close-Einheiten messen kann. Die Abbildung 2 stellt die Heterogenität dieses Enzyms in
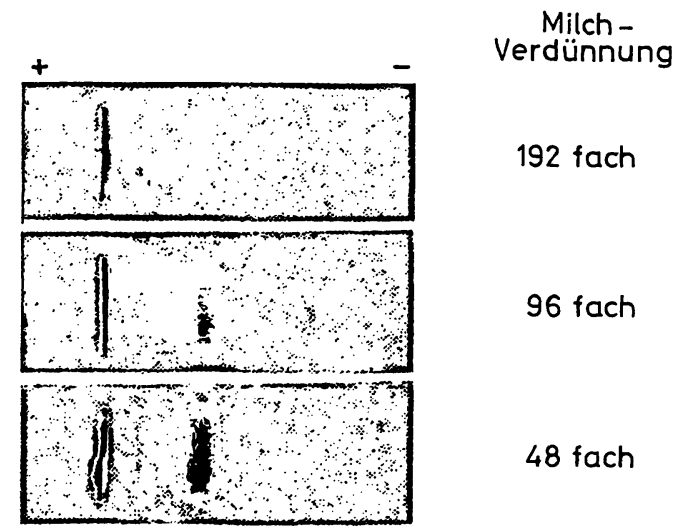

96 fach

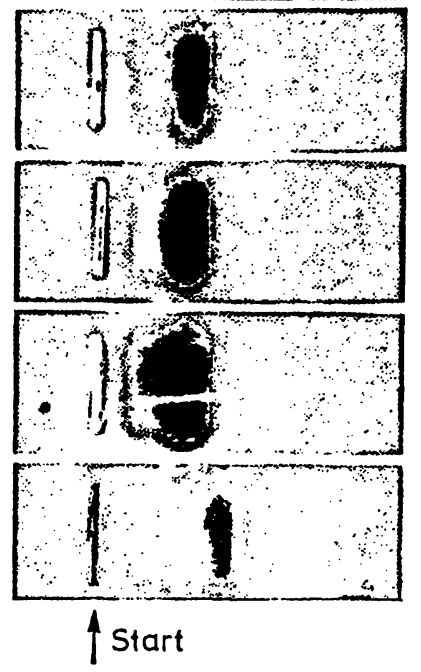

48 fach

24 fach

12 fach

6 fach

Abb. 2

Die Heterogenität der Amylase in Muttermilch. Verschiedene Verdünnungen einer einzelnen Milchprobe. Die Amylase-Aktivität dieser Probe beträgt $1720 \mathrm{E}$. nach STREET u. CLOSE. Ganz unten ein Blutserum-Enzymogramm

Muttermilch dar. Die einzelnen Enzymelektropherogramme zeigen verschiedene Verdünnungen einer einzelnen Probe. Das Zymogram der Blutserum-Amylase ist zum besseren Vergleich der elektrophoretischen Beweglichkeiten zugefügt. Bei der geringen Magensaft- 
azidität, besonders bei den Neugeborenen, kann man das Vorkommen der Amylase in Muttermilch als Substitutions- oder auch Induktions-Wirkung ansehen, wenn man an die Maltase des Dünndarmes denkt. Dagegen sind kaum Aktivitäten der Amylase in den untersuchten Sorten von Trockenmilch nachweisbar. Auch in der frischen, nicht pasteurisierten Kuhmilch ist die Amylase-Aktivität ganz gering. Die hohe Amylase-Aktivität in der nativen Muttermilch beweist den hohen biologischen Wert der Muttermilch.

Aus unserem Beitrag geht hervor, daß die einfache Bestimmung der Speichel- und Parikreas-Amylase im Blutserum der Kinder im Laufe des ersten Lebensjahres nicht nur genetische Gesichtspunkte, sondern auch Fragen, welche die Ernährung der Säuglinge berühren, präzisiert.

\section{Literatur}

1. KamarÝr, J., und R. Laxovi, Humangenetik 1, 579 (1965). 2. KAMarúr, J., Vnitřni lékařstvi 15, 239 (1969). - 3. KaMarÝr, J., diese Z. 7, 51 (1969). - 4. KamarłT, J., und R. Laxoví, Humangenetik 3, 41 (1966). - 5. LAXovi, R., und J. KaMarÝt, Febs, Proceedings of the Fifth Meeting, Prague, July 1968, Enzymes and Isoenzymes, Acad. Press 18, 335 (1970), London und
New York. - 6. Street, H. V., und J. R. Close, Clin. Chim. Acta, Amsterdam 1, 256 (1956). - 7. KLEINBAUM, H., Z. Kinderheilkunde 90, 7 (1964). - 8. KamarÝt, J., Čsl. dermatologie 43, 5 (1968). - 9. Halprin, K. M., und A. OhKawara, J. Investigat. Dermat. 46, 43 (1969).
RNDr. J. Kamarýt, C. Sc. Cernopoln 9

Brno, Tschechoslowakei 